\title{
SOME DIRECT AND INVERSE THEOREMS IN APPROXIMATION OF FUNCTIONS
}

\author{
R. N. MOHAPATRA and D. C. RUSSELL
}

(Received 25 February 1980; revised 12 May 1981)

Communicated by E. Strzelecki

\begin{abstract}
The paper is concerned with the determination of the degree of convergence of a sequence of linear operators connected with the Fourier series of a function of class $L_{p}(p>1)$ to that function and some inverse results in relating the convergence to the classes of functions. In certain cases one can obtain the saturation results too. In all cases $L_{p}$ norm is used.
\end{abstract}

1980 Mathematics subject classification (Amer. Math. Soc.): 41 A 40.

Let $f(x)$ be a periodic, Lebesgue integrable function with period $2 \pi$. Let the Fourier series for $f(x)$ be given by

$$
\frac{1}{2} a_{0}+\sum_{k=1}^{\infty}\left(a_{k} \cos k x+b_{k} \sin k x\right) \equiv \sum_{k=0}^{\infty} A_{k}(x) .
$$

Let $S_{n}(f ; x)$ be the $n$th partial sum of the series (1). The conjugate series of the series (1) is

$$
\sum_{n=1}^{\infty} B_{n}(x)=\sum_{k=1}^{\infty}\left(b_{k} \cos k x-a_{k} \sin k x\right) .
$$

The conjugate function $\tilde{f}$ of $f$, is given by

$$
\tilde{f}(x)=(2 \pi)^{-1} \int_{0}^{\pi}\{f(x+t)-f(x-t)\} \cot \frac{t}{2} d t
$$

the integral being interpreted as a Cauchy integral. It is known that $\tilde{f}$ exists almost everywhere whenever $f$ is integrable.

c. Copyright Australian Mathematical Society 1983 
The space $L_{p}[-\pi, \pi]$ when $p=\infty$ will be replaced by the space $c_{2 \pi}$ of all continuous functions defined over $[-\pi, \pi]$. Throughout the paper, norms will be taken with respect to the variable $x$ and $\|\cdot\|_{p}$ will denote the usual $L_{p}$ norm for $1<p<\infty$, and the supremum norm when $p=\infty$. For $f \in L_{p}[-\pi, \pi](1<p<$ $\infty)$, the modulus of continuity and the modulus of smoothness $w^{(p)}(\delta, f)$ and $w_{2}^{(\rho)}(\delta ; f)$ are defined respectively by

$$
\begin{gathered}
w^{(p)}(\delta ; f)=\sup _{|h| \leqslant \delta}\|f(x+h)-f(x)\|_{p}, \quad \text { and } \\
w_{2}^{(p)}(\delta ; f)=\sup _{|h| \leqslant \delta}\|f(x+h)+f(x-h)-2 f(x)\|_{p} .
\end{gathered}
$$

The classes $\operatorname{Lip} \alpha, \operatorname{Lip}(\alpha, p)(p \geqslant 1)$ will be as usual (see [5], page 612; also see [18], pages 42, 45). The class $\operatorname{Lip}(\alpha, p)$ with $p=\infty$ will be taken as $\operatorname{Lip} \alpha$.

Two functions $f$ and $g$ are said to be equivalent if $f(x)=g(x)$ almost everywhere.

Let $\left\{c_{n}\right\},\left\{d_{n}\right\}$ be two non-zero sequences with $c_{n}, d_{n} \geqslant 0$. Suppose $C_{n}=\sum_{k=0}^{n} c_{k}$ and $D_{n}=\sum_{k=0}^{n} d_{k}$. Let $R_{n}=c_{0} d_{n}+c_{1} d_{n-1}+\cdots+c_{n} d_{0}(n=0,1, \ldots)$.

Given $f$, let us associate with it the operator $t_{n}(f)$ defined by

$$
t_{n}(f ; x)=\left(R_{n}\right)^{-1} \sum_{k=1}^{n} c_{n-k} d_{k} S_{k}(x) .
$$

It should be remarked that $t_{n}(f ; x)$ is the $(N, c, d)$ transform of $\left\{S_{k}(f ; x)\right\}$ (see [2]).

We shall write $t_{n}(f ; x)=N_{n}(f ; x)$ or $\bar{N}_{n}(f ; x)$ according as $d_{n}=1$ for all $n$ or $c_{n}=1$ for all $n$.

If there exists a positive non-increasing function $\phi(n)$ and a normed linear space $K$ of functions such that

$$
\begin{gathered}
\left\|f(x)-t_{n}(f ; x)\right\|=o(\phi(n)) \Rightarrow f \text { is a constant a.e., } \\
\left\|f(x)-t_{n}(f ; x)\right\|=O(\phi(n)) \Rightarrow f \in K, \quad \text { and } \\
f \in K \Rightarrow\left\|f(x)-t_{n}(f ; x)\right\|=O(\phi(n))
\end{gathered}
$$

then we say that the operator $t_{n}(f)$ or the corresponding method $(N, c, d)$ is saturated with order $\phi(n)$ and class $K$.

Ever since the definition of saturation of summability methods was given by Favard [3] many authors have studied the saturation property of operators which are obtained as transforms of the $n$th partial sum of the Fourier series by 
summability methods. Sunouchi and Watari [15], [16] have obtained the saturation order and class for Cesàro, Abel and the Riesz method $\left(R, n^{\xi}, 1\right)(\zeta=$ $1,2, \ldots)$. Mohapatra and Sahney [11] have obtained results on saturation for a general class of summability methods in the supremum norm. Sunouchi [14] has studied the local saturation properties of the convolution operator (also see [13], [17]).

Concerning the saturation property of the Nörlund method, Goel, Holland, Nasim and Sahney [4] have proved the following theorem:

THEOREM A ([4], compare [9]). Let $f \in c_{2 \pi}$ and $C_{n}>0$ (all $n$ ). Then the following hold:

$$
\begin{aligned}
& \left\|f-N_{n}(f)\right\|_{\infty}=o\left(\frac{c_{n}}{C_{n}}\right) \Rightarrow f \text { is a constant a.e. } \\
& \left\|f-N_{n}(f)\right\|_{\infty}=o\left(\frac{c_{n}}{C_{n}}\right) \Rightarrow f \in\{f \mid \tilde{f} \in \operatorname{Lip~} 1\}
\end{aligned}
$$

whenever

$$
\begin{aligned}
& \lim _{n \rightarrow \infty} \frac{c_{n-k}}{c_{n}}=1 \quad\left(k=0,1, \ldots ; c_{n}>0 \text { for all } n\right) . \\
& f \in\{f \mid \tilde{f} \in \operatorname{Lip} 1) \Rightarrow\left\|f-N_{n}(f)\right\|_{\infty}=O\left(\frac{c_{n}}{C_{n}}\right)
\end{aligned}
$$

whenever

$$
\sum_{k=0}^{n}\left|c_{k}-c_{k-1}\right|=O\left(c_{n}\right) \quad\left(c_{-1}=0\right)
$$

In Section 3 we obtain the order and class of saturation of the method $(N, c, d)$ or the operator $t_{n}(f)$ in the $L_{p}(1<p \leqslant \infty)$ norm. Special cases of this result extend Theorem $\mathrm{A}$ and yield a saturation result for a type of Riesz method.

The other object of this paper is to obtain the degree of convergence of $t_{n}(f)$ to $f \in L_{p}$ in terms of the integral modulus of continuity and integral modulus of smoothness with a view to generalizing the following results:

THEOREM B ([12]). If $f \in \operatorname{Lip}(\alpha, p)\left(0<\alpha \leqslant 1, p>1, p^{-1}+p^{\prime-1}=1\right)$ and if $C_{n} \rightarrow \infty$ and

$$
\left(\int_{1}^{n} \frac{C(y)}{y^{p^{\prime} \alpha+2-p^{\prime}}} d y\right)^{1 / p^{\prime}}=O\left(C_{n} / n^{\alpha-p^{-1}}\right)
$$

(where $\left.C(y)=C_{[y]}\right)$ then

$$
\left\|f-N_{n}(f)\right\|_{p}=O\left(n^{-\alpha+p^{-1}}\right) .
$$


THEOREM C ([10]). Let $C_{n} \rightarrow \infty$ as $n \rightarrow \infty$, and $R(y) / y^{\alpha}$ be nondecreasing where $R(y)=R_{[y]}$. Then $f \in \operatorname{Lip}(\alpha, p)(0<\alpha<1, p>1)$ implies

$$
\left\|f-t_{n}(f)\right\|_{p}=O\left(n^{-\alpha+p^{-1}}\right) .
$$

THEOREM D ([8]). If $w(t)$ is the modulus of continuity of $f \in C[-\pi, \pi]$ and $c_{n}>0$, $c_{n} / C_{n}=O\left(n^{-1}\right)$,

$$
\left\|f-N_{n}(f)\right\|_{\infty}=O\left(\frac{1}{C_{n}} \sum_{k=1}^{n} \frac{w(1 / k)}{k} C_{k}\right) .
$$

In Section 4 we shall generalize these results and obtain some other special cases.

Following the method of Sunouchi and Watari [16] we can obtain

THEOREM 1. Let $1 \leqslant p \leqslant \infty$. The following hold:

$$
\left\|f-t_{n}(f)\right\|_{p}=o\left(\frac{c_{n}}{R_{n}}\right) \Rightarrow f \text { is equivalent to a constant. }
$$

When $c_{n-k} / c_{n} \rightarrow 1$ as $n \rightarrow \infty$, $k$ fixed, we have

$$
\left\|f-t_{n}(f)\right\|_{p}=O\left(\frac{c_{n}}{R_{n}}\right) \Rightarrow\left\|\sum_{k=1}^{N} D_{k-1}\left(1-\frac{k}{N+1}\right) A_{k}(x)\right\|_{p}=O(1) .
$$

Thus $\left\|f-t_{n}(f)\right\|_{p}=O\left(c_{n} / R_{n}\right)$ implies

(20) $\sum_{k=1}^{\infty} D_{k-1} A_{k}(x)$ is the Fourier series of a bounded function, when $p=\infty$;

$$
\begin{gathered}
\sum_{k=1}^{\infty} D_{k-1} A_{k}(x) \text { is the Fourier series of a function of class } L_{p}, \\
\quad \text { when } 1<p<\infty ; \\
\sum_{k=1}^{\infty} D_{k-1} A_{k}(x) \text { is the Fourier-Stieltjes series of a function } \\
\text { of bounded variation, when } p=1 .
\end{gathered}
$$

Throughout the paper, we write for $1 \leqslant p<\infty, K_{p}=\left\{f \in L_{p} \mid \tilde{f} \in \operatorname{Lip}(1, p)\right\}$, and $K_{\infty}=\left\{f \in c_{2 \pi} \mid f \in \operatorname{Lip} 1\right\}$. 
If $d_{n}=1$ for all $n$, then we have, from Theorem 1:

Corollary 1. Let $C_{n}>0($ all $n)$. Then

$$
\left\|f-N_{n}(f)\right\|_{p}=o\left(c_{n} / C_{n}\right) \Rightarrow f \text { is equivalent to a constant, }
$$

and if (11) holds then

$$
\left\|f-N_{n}(f)\right\|_{p}=O\left(c_{n} / C_{n}\right) \Rightarrow f \in K_{p} \quad(1 \leqslant p \leqslant \infty) .
$$

Proof. It is enough to deduce (24). When (11) holds we observe that the conclusion in (19) shows that the $(C, 1)$ mean of $\sum_{k=1}^{\infty} k A_{k}(x)$ is uniformly bounded in the $L_{p}$ norm $(1 \leqslant p \leqslant \infty)$. Since $-\Sigma_{k} k A_{k}(x)=\Sigma_{k} B_{k}^{\prime}(x)$ where $\sum B_{k}(x)$ is the conjugate series of the Fourier series of $f(x)$, we have $\left\|\sigma_{N}^{\prime}\right\|_{p}=O(1)$ where $\sigma_{N}(x)$ is the first Cesàro mean of $\Sigma B_{k}$. This is known to be equivalent to $f \in K_{p}$.

Remarks. 1. If $p>1$, then the conclusion $f \in K_{p}$ in Corollary 1 can be replaced by $f \in \operatorname{Lip}(1, p)$ (see [6], Lemma 13, page 621).

2. (20), (21) and (22) refer to the Fourier series $\sum_{k=1}^{\infty} D_{k-1} A_{k}(x)$. Since we do not know much about the behaviour of that series the saturation problem for $(\bar{N}, d)$ turns out to be difficult. However when $p=2$ we get the following as an easy consequence of Parseval's identity:

COROLlaRy 2. Let $f \in L_{2}$. Corresponding to the order of saturation $1 / D_{n}$ the saturation class of the method $(\bar{N}, d)$ or of the operator $\vec{N}_{n}(f)$ is the class of all functions $f \in L_{2}$ with Fourier series $\sum_{k=1}^{\infty} D_{k-1} A_{k}(x)$.

Our next result gives an estimate for the error in approximating a function $f \in K_{p}$ by $t_{n}(f)$. Precisely, we prove

THEOREM 2. Let $1<p \leqslant \infty$ and $\left\{c_{n}\right\}$ and $\left\{d_{n}\right\}$ satisfy

$$
\sum_{k=0}^{n}\left|c_{n-k} d_{k}-c_{n-k-1} d_{k+1}\right|=O\left(c_{n}\right) \text {. }
$$

Then, for $f \in K_{p}$,

$$
\left\|f-t_{n}(f)\right\|_{p}=O\left(c_{n} / R_{n}\right)
$$

We shall need the following lemmas for the proof of our theorem:

LeMma 1 ([5], Theorem 24(i), page 599). If $f$ belongs to $\operatorname{Lip}(1, p)(1<p \leqslant \infty)$ then $f$ is equivalent to the indefinite integral of a function belonging to $L_{p}$. If $f \in \operatorname{Lip} 1$ then $f$ is the indefinite integral of a bounded function. 
Lemma 2 ([6], Theorem 5, page 627). Suppose $f \in \operatorname{Lip}(\alpha, p)$ where $p \geqslant 1,0<\alpha$ $\leqslant 1$.

(i) If $\alpha p \leqslant 1$ and $p<q<p /(1-\alpha p)$, then $f \in \operatorname{Lip}(\alpha-1 / p+1 / q, q)$.

(ii) If $\alpha p>1$ then $f \in \operatorname{Lip}(\alpha-1 / p+1 / q, q)$ for all $q>p$, and $f$ is equivalent to a function of $\operatorname{Lip}(\alpha-1 / p)$.

LEMMA 3. Let

$$
K_{n}(t)=\left(R_{n}\right)^{-1} \sum_{k=1}^{n} c_{n-k} d_{k} \frac{\cos (k+1 / 2) t}{\sin t / 2},
$$

and

$$
L_{n}(t)=\int_{t}^{\pi} K_{n}(u) d u
$$

Then

$$
\begin{array}{r}
f \in K_{p}(1<p \leqslant \infty) \text { implies }\left\|f-t_{n}(f)\right\|_{p}=O\left(c_{n} / R_{n}\right) \\
\text { if } \int_{0}^{\pi}\left|L_{n}(t)\right| d t=O\left(c_{n} / R_{n}\right) .
\end{array}
$$

Proof. Let $\tilde{S}_{n}(\tilde{f} ; x)$ denote the partial sums of the conjugate series associated with $\tilde{f}(x)$. We have, from the definition,

$$
\begin{aligned}
& t_{n}\left(\tilde{S_{n}}(\tilde{f} ; x)\right)=\left(2 \pi R_{n}\right)^{-1} \sum_{k=0}^{n} c_{n-k} d_{k} \int_{0}^{\pi}[\tilde{f}(x+t)-\tilde{f}(x-t)] \cot \frac{t}{2} d t \\
& -\left(2 \pi R_{n}\right)^{-1} \sum_{k=n}^{n} c_{n-k} d_{k} \int_{0}^{\pi}[\tilde{f}(x+t)-\tilde{f}(x-t)] \cos \left(k+\frac{1}{2}\right) t \csc \frac{t}{2} d t
\end{aligned}
$$

By M. Riesz's theorem (Zygmund [18], Theorem (2.4),page 253) $f \in L_{p}(1<p$ $<\infty) \Rightarrow \tilde{f} \in L_{p} \Rightarrow \tilde{f} \in L_{p}$ and $\tilde{S}(\tilde{f})=S(\tilde{f})$. If $p=\infty, \tilde{f} \in$ Lip 1 (by hypothesis) and then $-f+\frac{1}{2} a_{0}$ is identical to $\tilde{\tilde{f}}$. Thus from (30) and (27),

$$
f(x)-t_{n}(f ; x)=(2 \pi)^{-1} \int_{0}^{\pi}\{\tilde{f}(x+t)-\tilde{f}(x-t)\} K_{n}(t) d t
$$

almost everywhere.

Since $f \in K_{p}$, by Lemma 1 , we can take $\tilde{f}(u)$ equivalent to the indefinite integral of a function, say $\tilde{f}^{\prime}(u) \in L_{p}(p>1)$. By integration by parts, we have from (31)

$$
f(x)-t_{n}(f ; x)=(2 \pi)^{-1} \int_{0}^{\pi}\left\{\tilde{f}^{\prime}(x+t)+\tilde{f}^{\prime}(x-t)\right\} L_{n}(t) d t .
$$


By using the generalized Minkowski's inequality ([7], page 148, 6.13.9)

$$
\begin{aligned}
\left\|f-t_{n}(f)\right\|_{p} & \leqslant(2 \pi)^{-1} \int_{0}^{\pi}\left\|\tilde{f}^{\prime}(x+t)+\tilde{f}^{\prime}(x-t)\right\|_{p}\left|L_{n}(t)\right| d t \\
& =O\left(\int_{0}^{\pi}\left|L_{n}(t)\right| d t\right)=O\left(c_{n} / R_{n}\right) .
\end{aligned}
$$

LEMMA 4 ([4]).

$$
\begin{aligned}
& \left|\int_{t}^{\pi} \frac{\sin (k+1) u}{u^{2}} d u\right| \\
& \quad \leqslant \begin{cases}2(k+1) \log \frac{1}{(k+1) t} & \text { for } 0<(k+1) t<1 / e ; \\
2 /(k+1) t^{2} & \text { for any } k \geqslant 0, t>0 .\end{cases}
\end{aligned}
$$

The lemma can be proved easily.

Proof of Theorem 2. In view of Lemma 3, it is enough to prove (29). By Abel's transformation

$$
-K_{n}(t)=\left(2 R_{n} \sin ^{2} \frac{t}{2}\right)^{-1} \sum_{k=0}^{n}\left(c_{n-k} d_{k}-c_{n-k-1} d_{k+1}\right) \sin (k+1) t .
$$

Since

$$
\left(2 \sin ^{2} \frac{t}{2}\right)^{-1}=\frac{2}{t^{2}}+O(1)
$$

we get, from (33) and (25), that

$$
-K_{n}(t)=\left(2 / R_{n} t^{2}\right), \sum_{k=0}^{n}\left(c_{n-k} d_{k}-c_{n-k-1} d_{k+1}\right) \sin (k+1) t+O\left(c_{n} / R_{n}\right) .
$$

From (33), we observe that (29) holds if

$$
\sum_{k=0}^{n}\left|\left(c_{n-k} d_{k}-c_{n-k-1} d_{k+1}\right)\right| \int_{0}^{\pi}\left|\int_{t}^{\pi} \frac{\sin (k+1) u}{u^{2}} d u\right| d t=O(1) .
$$

In view of (25), (34) is true whenever

$$
\int_{0}^{\pi}\left|\int_{t}^{\pi} \frac{\sin (k+1) u}{u^{2}} d u\right| d t=O(1)
$$

uniformly in $k$. 
By Lemma 4, the integral on the left of (35) does not exceed

$$
\begin{aligned}
\int_{0}^{1 / e(k+1)} & \left|\int_{t}^{\pi} \frac{\sin (k+1) u}{u^{2}} d u\right| d t+\int_{1 / e(k+1)}^{\pi}\left|\int_{t}^{\pi} \frac{\sin (k+1) u}{u^{2}} d u\right| d t \\
& \leqslant \int_{0}^{1 / e(k+1)} 2 \log (1 /(k+1) t) d t+\int_{1 / e(k+1)}^{\pi} 2(k+1)^{-1} t^{-2} d t
\end{aligned}
$$

Since each integral is bounded the result follows.

Corollary 3. Let $\left\{d_{n}\right\} \in b v, d_{n} \geqslant 0, D_{n}>0$. If $f \in K_{p}(1<p \leqslant \infty)$, then

$$
\left\|f-\bar{N}_{n}(f)\right\|_{p}=O\left(D_{n}^{-1}\right) \text {. }
$$

COROLlaRY 4. Let $\left\{c_{n}\right\}$ satisfy $C_{n} \geqslant 0, C_{n}>0$, and

$$
\sum_{k=0}^{n}\left|c_{k}-c_{k-1}\right|=O\left(c_{n}\right) \quad\left(c_{-1}=0\right) .
$$

Then $f \in K_{p}$ implies $\left\|f-N_{n}(f)\right\|_{p}=O\left(c_{n} / C_{n}\right)(1<p \leqslant \infty)$.

The case $p=\infty$ is given in [4, Lemma 2.3].

Combining Corollary 1 and Corollary 4 , we get the following:

THEOREM 3. Let $\left\{c_{n}\right\}$ satisfy (11) and (36). Then the Nörlund method $\left(N, c_{n}\right)$ is saturated with order $c_{n} / C_{n}$ and class $K_{p}$.

REMARK. Lemma 3 shows that (29) is a sufficient condition for $\left\|f-t_{n}(f)\right\|_{p}$ $=O\left(c_{n} / R_{n}\right)$ whenever $f \in K_{p}(1<p \leqslant \infty)$. We do not know if (29) is also necessary.

\section{4}

Let us write $R(y)=R_{[y]}$. With a view to generalizing Theorem B and Theorem $\mathrm{C}$, and extending Theorem $\mathrm{D}$, we prove the following:

THEOREM 4. Let $\left\{c_{n}\right\},\left\{d_{n}\right\}$ be non-negative, non-increasing sequences and $R_{n}>0$. Let $f \in L_{p}[-\pi, \pi](1<p<\infty)$ or $f \in c_{2 \pi}(p=\infty)$. Then

$$
\left\|f-t_{n}(f)\right\|_{p}=O\left(\frac{1}{R_{n}} \sum_{k=1}^{n} \frac{w_{2}^{(p)}(1 / k)}{k} R_{k}\right)+o\left(w_{2}^{(p)}\left(\frac{1}{n}\right)\right) .
$$


REMARK. If in addition to the hypotheses assumed on the sequences $\left\{c_{n}\right\}$ and $\left\{d_{n}\right\}$, we assume that there exists $l>0$ such that

$$
\left(R_{n}\right)^{-1} \sum_{k=1}^{n}\left(R_{k} / k\right) \geqslant l \quad(n=1,2, \ldots)
$$

then

$$
w_{2}^{(p)}\left(\frac{1}{n}\right) \leqslant\left(l R_{n}\right)^{-1} \sum_{k=1}^{n}\left\{\frac{R_{k} w_{2}^{(p)}(1 / k)}{k}\right\} .
$$

Hence we can get from (42) that

$$
\left\|f-t_{n}(f)\right\|_{p}=O\left(\frac{1}{R_{n}} \sum_{k=1}^{n}\left(\frac{R_{k} w_{2}^{(p)}(1 / k)}{k}\right)\right) .
$$

We shall need the following lemma for the proof of our theorem.

LEMMA 5 ([10]). If $\left\{c_{n}\right\}$ and $\left\{d_{n}\right\}$ are non-negative, non-increasing sequences and $\tau=[1 / t]$ then for $0 \leqslant a<b \leqslant n($ any $n)$, and $0<|t| \leqslant \pi$, we have

$$
\left|\sum_{k=1}^{b} c_{n-k} d_{k} \sin k t\right|=O(R(\tau)) \text { as } t \rightarrow 0 .
$$

Proof of Theorem 4. We easily get

$$
f(x)-t_{n}(f ; x)=\int_{0}^{\pi}\{f(x+t)+f(x-t)-2 f(x)\} M_{n}(t) d t
$$

where

$$
M_{n}(t)=\left(2 \pi R_{n}\right)^{-1} \sum_{k=0}^{n} c_{n-k} d_{k} \frac{\sin \left(k+\frac{1}{2}\right) t}{\sin t / 2} .
$$

Hence, by generalized Minkowski's inequality

$$
\left\|f(x)-t_{n}(f ; x)\right\| \leqslant I_{1}+I_{2},
$$

where

$$
I_{1}=\int_{0}^{\pi / n} w_{2}^{(p)}(t ; f)\left|M_{n}(t)\right| d t, \quad \text { and } \quad I_{2}=\int_{\pi / n}^{\pi} w_{2}^{(p)}(t ; f)\left|M_{n}(t)\right| d t .
$$

Since $0<\sin \left(k+\frac{1}{2}\right) t<(2 k+1) \sin t / 2$ for $0 \leqslant k \leqslant n, 0<t<\pi / n$, we have

$$
I_{1}=O\left((2 n+1) \int_{0}^{\pi / n} w_{2}^{(p)}(t ; f) d t\right)=O\left(w_{2}^{(p)}\left(\frac{\pi}{n} ; f\right)\right) .
$$


By Lemma 5,

$$
\begin{aligned}
I_{2} & =O\left(\frac{1}{R_{n}} \int_{\pi / n}^{\pi} \frac{R(1 / t)}{t} w_{2}^{(p)}(t ; f) d t\right) \\
& =O\left(\frac{1}{R_{n}} \sum_{k=1}^{n-1} \int_{k / \pi}^{(k+1) / \pi} \frac{w_{2}^{(p)}\left(\frac{1}{t}, f\right) R(t)}{t} d t\right) \\
& =O\left(\frac{1}{R_{n}} \sum_{k=1}^{n}\left(\frac{R_{k} w_{2}^{(p)}(1 / k)}{k}\right)\right)
\end{aligned}
$$

On collecting the estimates, the theorem follows.

REMARK. If $0<\alpha \leqslant 1, p>1, \alpha p>1$ then, by Lemma 2(ii), $f \in \operatorname{Lip}(\alpha, p)$ implies $w_{2}^{(p)}(\delta ; f)=O\left(\delta^{\alpha-1 / p}\right)$. In this case

$$
w_{2}^{(p)}(1 / n)=O\left(n^{-\alpha+1 / p}\right)
$$

and

$$
\frac{1}{R_{n}} \sum_{k=1}^{n} \frac{R_{k} w_{2}^{(p)}(1 / k)}{k}=O\left(\frac{1}{R_{n}} \sum_{k=1}^{n} R_{k} \frac{1}{k^{\alpha+1-1 / p}}\right)
$$

Let $\delta \geqslant 0$ and $A_{n}^{\delta}$ be given by $\sum_{n=0}^{\infty} A_{n}^{\delta} x^{n}=(1-x)^{-\delta-1}(|x|<1)$. Let $N_{n}(f)$ be written as $\sigma_{n}^{\delta}(f)$ or $H_{n}(f)$ according as $c_{n}=A_{n}^{\delta-1}$ or $c_{n}=(n+1)^{-1}$ for all $n$.

By putting $d_{n}=1$ for all $n$, we get the following results:

Corollary 5. Let $f \in \operatorname{Lip}(\alpha, p), 1<p \leqslant \infty$. Then

$$
\left\|f-\boldsymbol{\sigma}_{n}^{\delta}(f)\right\|_{p}= \begin{cases}O\left(n^{-\delta+1 / p}\right) & (0<\delta<\alpha \leqslant 1) ; \\ o\left(\frac{\log n}{n^{\delta-1 / p}}\right) & (0<\delta \leqslant \alpha \leqslant 1) .\end{cases}
$$

Remark. The case $p=\infty$ of Corollary 5 was proved by Alexits [1].

COROLlaRY 6. If $\left\{c_{n}\right\}$ is a positive non-increasing sequence and $f \in L_{p}[-\pi, \pi]$ $(1<p<\infty)$ or $f \in c_{2 \pi}(p=\infty)$, then

$$
\left\|f-N_{n}(f)\right\|_{p}=O\left(\frac{1}{C_{n}} \sum_{k=1}^{n} \frac{C_{k}}{k} w^{(p)}\left(\frac{1}{k} ; f\right)\right) .
$$

Remark. The case $p=\infty$ of this Corollary is Theorem D. 
Corollary 7. If $f \in \operatorname{Lip}(\alpha, p), \alpha p>1,0<\alpha \leqslant 1, p>1$, then

$$
\left\|f-H_{n}(f)\right\|_{p}=O\left((\log n)^{-1}\right) \text {. }
$$

In what follows, we shall write $\bar{H}_{n}(f)$ for $\bar{N}_{n}(f)$ when $d_{n}=1 /(n+1)$.

COROLlaRY 8. Let $\left\{d_{n}\right\}$ be a non-negative, non-increasing sequence. Then for $f \in L_{p}[-\pi, \pi](1<p<\infty)$, or $f \in c_{2 \pi}(p=\infty)$,

$$
\left\|f-\bar{N}_{n}(f)\right\|_{p}=O\left(w^{(p)}(1 / n)\right)+o\left(\frac{1}{D_{n}} \sum_{k=1}^{n} \frac{D_{k} w^{(p)}(1 / k)}{k}\right) .
$$

CoRollary 9. If $f \in \operatorname{Lip}(\alpha, p), \alpha p>1,0<\alpha \leqslant 1, p>1$, then

$$
\left\|f-\bar{H}_{n}(f)\right\|_{p}=O\left((\log n)^{-1}\right) \text {. }
$$

Remarks. (i) Since $w_{2}^{(p)}(\delta, f) \leqslant 2 w^{(p)}(\delta, f)$, Corollary 6 and Corollary 8 are stated with estimates using modulus of continuity in place of integral modulus of smoothness.

(ii) It can be observed that our corollaries contain assumptions on $\left\{c_{n}\right\}$ and $\left\{d_{n}\right\}$ but we do not use conditions of the type (14) (see Theorem B and Theorem C).

Finally we are grateful to the referee for his valuable comments.

\section{References}

[1] G. Alexits, 'Über die Annäherung einer stetigen Funktion durch die Cesàroschen Mittel ihrer Fourrierreihe', Math. Ann. 100 (1928), 264-277.

[2] D. Borwein, 'On products of sequences', J. London Math. Soc. 33 (1958), 212-220.

[3] J. Favard, 'Sur la saturation des procédés de sommation', J. Math. Pures Appl. 36 (1957), 359-372.

[4] D. S. Goel, A. S. B. Holland, C. Nasim and B. N. Sahney, 'Best approximation by a saturation class of polynomial operators', Pacific J. Math. 55 (1974), 149-155.

[5] G. H. Hardy and J. E. Littlewood, 'Some properties of fractional integrals I', Math. Z. 27 (1928), 565-600.

[6] G. H. Hardy and J. E. Littlewood, 'A convergence criterion for Fourier series', Math. Z. 28 (1928), 612-634.

[7] G. H. Hardy, J. E. Littlewood and G. Polya, Inequalities (Cambridge, 1934, 1967).

[8] A. S. B. Holland, B. N. Sahney and J. Tzimbalario, 'On the degree of approximation of a class of functions by Fourier Series', Acta. Sci. Math. (Szeged) 38 (1976), 69-72.

[9] H. H. Khan and S. M. Rizvi, 'On the saturation classes of functions by $\left(N, p_{n}, q_{n}\right)$ method', Indian J. Pure Appl. Math. 6 (1974), 1262-1269.

[10] H. H. Khan, 'On the degree of approximation of functions belonging to class $\operatorname{Lip}(\alpha, p)$ ', Indian J. Pure Appl. Math. 5 (1974), 132-136. 
[11] R. N. Mohapatra and B. N. Sahney, 'Saturation of a class of linear operators involving a lower-triangular matrix', Acta Sci. Math. (Szeged), to appear.

[12] B. N. Sahney and V. G. Rao, 'Error bounds in the approximation of functions ', Bull.Austral. Math. Soc. 6 (1972), 11-18.

[13] G. Sunouchi, 'On the class of saturation in the theory of approximation II, III ',Tôhoku Math. $J .13$ (1961), 112-118; 320-328.

[14] G. Sunouchi, 'Local saturation in convolution operators', to appear.

[15] G. Sunouchi and C. Watari, 'On determination of the class of saturation in the theory of approximation of functions', Proc. Japan Acad. 34 (1958), 477-481.

[16] G. Sunouchi and C. Watari, 'On determination of the class of saturation in the theory of approximation of functions II', Tôhoku Math. J. 11 (1959), 480-488.

[17] M. Zamanski, 'Classes de saturation de certains procédés d'approximation des séries de Fourier ', Ann. Sci. Ecole Norm. Sup. 66 (1949), 19-93.

[18] A. Zygmund, Trigonometric Series, Vol. I and Vol. II (Cambridge University Press, 1968).

Department of Mathematics

American University of Beirut

Beirut

Lebanon

\author{
Department of Mathematics \\ York University \\ Downsview, Ontario M3J 1P3 \\ Canada
}

